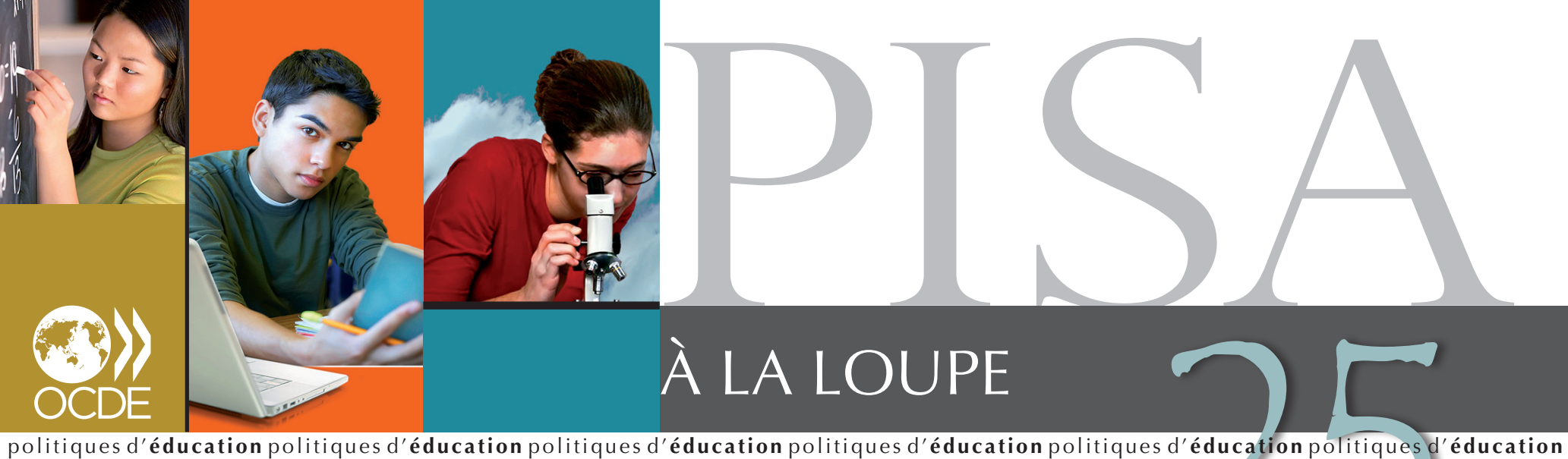

politiques d'éducation politiques d'éducation politiques d'éducation politiques d'éducation politiques d'éducation politiques d'éducation

\title{
Les pays s'orientent-ils vers des systèmes d'éducation plus équitables?
}

- Les résultats du PISA montrent qu'aucun pays ni économie n'est parvenu à créer un système d'éducation totalement équitable, même si certains sont bien plus avancés que d'autres dans ce domaine.

- Certains pays et économies ont prouvé qu'il est possible de progresser vers plus d'équité tout en améliorant la performance globale, et ce dans un délai relativement court.

L'enquête PISA montre de façon systématique l'existence d'une corrélation entre désavantage socio-économique et faible performance scolaire...
Dans l'idéal, les systèmes d'éducation sont censés offrir des possibilités d'apprentissage de qualité à l'ensemble de leurs élèves, indépendamment du milieu dont ces derniers sont issus. Les élèves venant de familles favorisées sur le plan socio-économique et ceux venant de familles défavorisées devraient donc bénéficier de chances égales de réussite à l'école - enfin, en théorie. Dans la plupart des pays, la réalité est en fait tout autre. Les résultats du PISA montrent de façon systématique l'existence d'une corrélation entre désavantage socio-économique et faible performance scolaire. Ainsi, en moyenne, dans les pays de l'OCDE, les élèves défavorisés sont deux fois plus susceptibles que les élèves favorisés d'appartenir au groupe des élèves les moins performants en compréhension de l'écrit. En moyenne, un élève issu d'un milieu socio-économique favorisé devance un élève issu d'un milieu socio-économique défavorisé de 88 points de score à l'évaluation PISA de la compréhension de l'écrit, soit un écart équivalant à plus de deux années de scolarité.

Toutefois, le fait que le degré de corrélation entre milieu socio-économique et rendement de l'apprentissage varie selon les pays et les économies prouve que l'origine sociale n'est pas une fatalité et que les politiques et les pratiques peuvent faire la différence. En outre, les pays peuvent œuvrer en faveur de l'équité du rendement de l'apprentissage tout en améliorant la performance des élèves. Dans l'enquête PISA 2009, nombre des pays et économies présentant le plus fort degré d'équité dans le rendement de l'apprentissage se classaient ainsi également parmi les plus performants. Au Canada, en Corée, en Estonie, en Finlande, à Hong-Kong (Chine), en Islande, au Liechtenstein et en Norvège, les élèves obtiennent des résultats supérieurs à la moyenne en compréhension de l'écrit et l'écart de performance entre élèves favorisés et élèves défavorisés est inférieur à 70 points de score. D'autres pays et économies sont également bien placés sur le plan de l'équité du rendement de l'apprentissage, mais leurs élèves ne sont pas aussi performants.

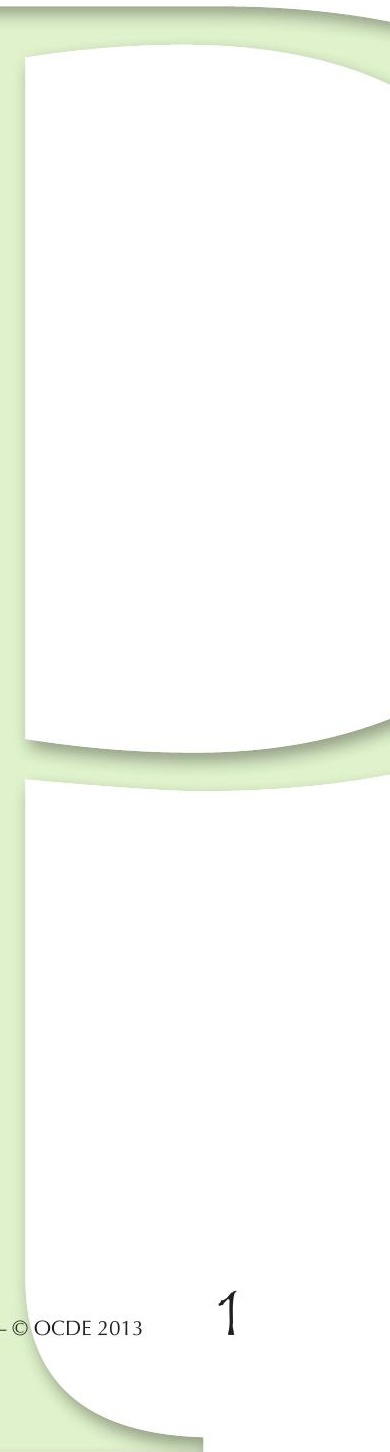


L'Azerbaïdjan, I'Indonésie, la Jordanie, Macao (Chine), le Qatar, la Serbie, la Thaïlande et la Tunisie offrent un niveau d'équité comparable à celui du groupe de pays précédent, mais leurs élèves obtiennent des résultats inférieurs à la moyenne de l'OCDE en compréhension de I'écrit. C'est en Allemagne, en Argentine, en Autriche, en Belgique, en Bulgarie, à Dubaï (EAU), aux États-Unis, en France, en Hongrie, en Israël, au Luxembourg, en Nouvelle-Zélande, au Panama, au Pérou et en Uruguay que l'écart de performance en compréhension de l'écrit entre élèves favorisés et élèves défavorisés est le plus marqué - supérieur à 100 points de score.

... mais il ne s'agit pas pour autant d'une fatalité.

Le degré de corrélation entre différences socio-économiques et écarts de performance varie selon les systèmes d'éducation, montrant ainsi que tout obstacle découlant d'un milieu socio-économique défavorisé peut être surmonté. Si certaines de ces variations peuvent être mises au compte de facteurs culturels, l'évolution significative de cette corrélation dans certains pays laisse penser que les politiques et les pratiques peuvent faire la différence.

\section{Équité et performance des pays/économies dans l'enquête PISA 2009}

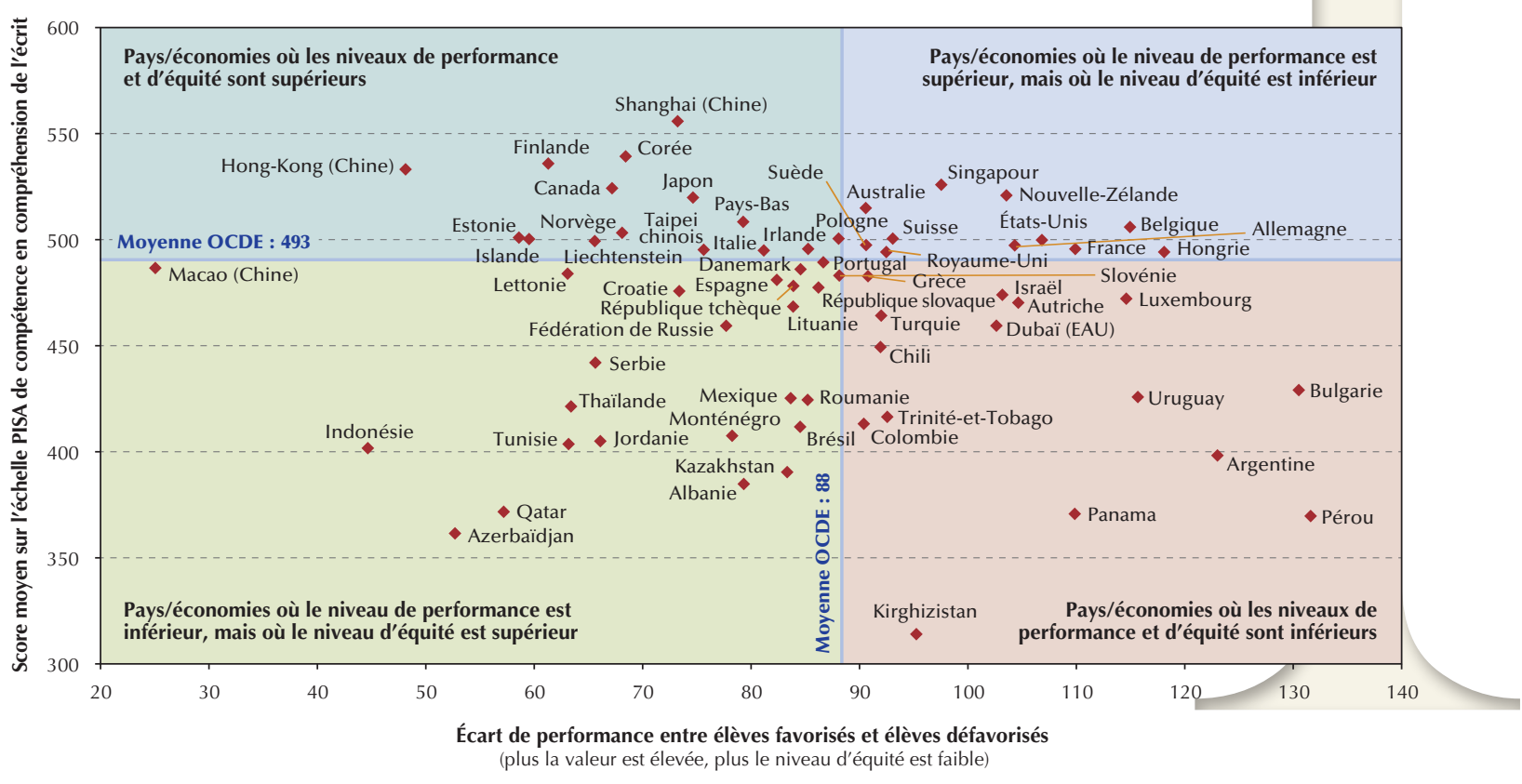

Sources : OCDE (2010), Résultats du PISA 2009 : Savoirs et savoir-faire des élèves, Volume I, Éditions OCDE, tableau I.2.3 ; et OCDE (2010), Résultats du PISA 2009 Surmonter le milieu social, Volume II, Éditions OCDE, tableau II.3.1.

De nombreux pays et économies ont réalisé d'importants progrès pour combler les écarts de performance entre élèves favorisés et élèves défavorisés, tout en parvenant simultanément à améliorer leur performance globale. Ce constat montre que les systèmes d'éducation sont en mesure de réduire le degré de corrélation entre les différences de milieu socio-économique et les résultats des élèves, tout en améliorant la performance globale de tous les élèves. Selon I'analyse comparative des résultats des enquêtes PISA 2000 et PISA 2009, en Albanie, en Allemagne, au Chili et en Lettonie, la corrélation entre le statut socio-économique des élèves et leurs résultats en compréhension de l'écrit s'est atténuée, en même temps que la performance globale des élèves en compréhension de l'écrit s'est améliorée. En Allemagne, par exemple, l'écart de performance entre élèves favorisés et élèves défavorisés s'est réduit de plus de 25 points de score et la performance moyenne en compréhension de l'écrit a progressé de 13 points de score. Au Chili, la performance moyenne en compréhension de l'écrit s'est améliorée de 40 points de score et l'écart de performance entre élèves favorisés et élèves défavorisés s'est réduit de plus de 15 points de score. 


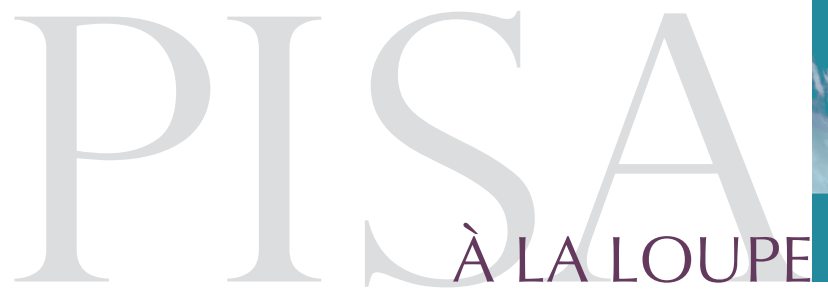

D'autres pays et économies - le Canada, les États-Unis, Hong-Kong (Chine), le Mexique et la République tchèque - ont également progressé sur la voie de l'équité, mais sans amélioration simultanée de leur performance globale.

\section{La mise en ourre de politiques adéquates peut aider à atténuer cette corrélation.}

En Albanie, en Allemagne, au Chili et en Lettonie, la corrélation entre le statut socio-économique des élèves et leur performance en compréhension de l'écrit s'est atténuée entre 2000 et 2009, tandis que la performance globale a progressé. L'équité et la performance se sont améliorées simultanément dans ces pays, soit parce que tous les élèves, quelle que soit leur origine socio-économique, ont progressé - avec l'amélioration la plus importante parmi les élèves défavorisés (par exemple, en Albanie et au Chili) -, soit parce que, même si la performance des élèves favorisés n'a pas évolué de façon significative, celle des élèves défavorisés a, quant à elle, été notable (par exemple, en Allemagne et en Lettonie).
Parmi les politiques d'éducation susceptibles de favoriser l'amélioration de l'équité et des performances, il est possible : d'offrir un soutien plus important et de meilleure qualité aux élèves défavorisés, qui débutent leur scolarité avec des lacunes en matière d'éducation; de garantir que tous les établissements d'enseignement proposent une instruction de qualité ; et d'offrir des possibilités $d^{\prime}$ apprentissage supplémentaires aux élèves défavorisés, leurs parents n'étant peut-être pas en mesure de le faire. Davantage de soutien pourrait être offert aux établissements d'enseignement présentant un effectif important d'élèves défavorisés ou aux élèves défavorisés au sein des établissements. Enfin, des politiques sociales plus larges permettant de garantir que les élèves favorisés et les élèves défavorisés n'ont pas des vécus trop différents, aussi bien à la maison - et tout particulièrement avant le début de la scolarité formelle -, qu'à l'école, peuvent également favoriser l'amélioration de l'équité comme des performances.

Évolution des niveaux d'équité et de performance entre 2000 et 2009

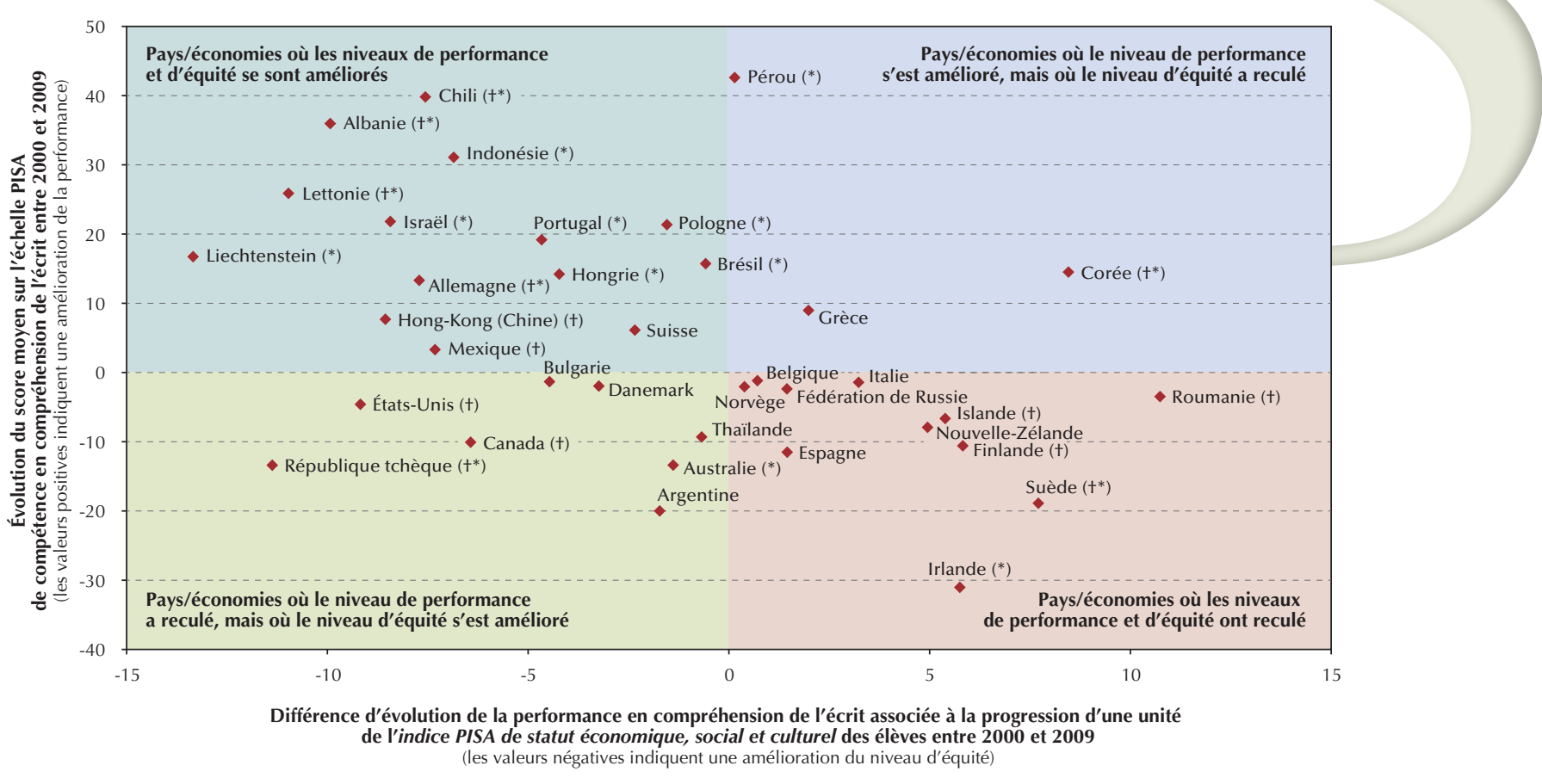

Remarque : on désigne en général l'évolution de la performance en compréhension de l'écrit associée à la progression d'une unité de l'indice PISA de statut économique, social et culturel des élèves comme la «pente du gradient socio-économique », qui correspond à la pente d'une régression du statut socio-économique sur la performance des élèves en compréhension de l'écrit. La différence de cette évolution entre 2009 et 2000 figure sur l'axe horizontal.

† L'évolution du niveau d'équité est statistiquement significative. * L'évolution du niveau de performance est statistiquement significative.

Source : OCDE (2010), Résultats du PISA 2009 : Tendances dans l'apprentissage, Volume V, Éditions OCDE, tableaux V.2.1 et V.4.3. 
